\title{
Microcomputer, minicomputer, mainframe interfacing
}

\author{
MICHAEL YOST, JAMES HOLCOMBE, and DOUGLAS EDDY \\ Trinity University, San Antonio, Texas
}

\begin{abstract}
The problems associated with the transfer of data from one computer to another in conducting neuropsychological research are described. The reasons for using different computers to perform different tasks are outlined, and a rationale for using task-specific computers is presented. A detailed description of the use and function of the Kermit File Transfer Protocol in neuropsychological research is given.
\end{abstract}

There are a number of different types of computer networks. The ultimate network is one in which there is conversation in both directions between any of the computers in the network. Since the ability to communicate between any of the computers is not always a requirement, then the ultimate network is not always necessary. Much of the communication between the computers in research laboratories is for the transfer of data files. This is true in the neuropsychology laboratory at Trinity University. As a part of our research, we transfer data from one computer to another. Analog data are collected from subjects with a PDP-11/24 computer. Once the data are collected and analog-to-digital (A/D) converted, they are sent to an IBM 370-3031 and submitted to a Fast Fourier Transform (FFT). These data are then sent to an IBM PC-XT, where they are statistically analyzed. On occasion, data are collected at a remote site and sent to the laboratory through a telephone line to one of our computers.

When the PDP-11/24 was installed, the hardware/software combination needed for high-speed, error-free file transfer between this computer and the IBM/370-3031 was not purchased. A transfer system of this type was not readily available, and if it had been, the cost would have exceeded our budget. The first communications between the two computers were through the use of a FORTRAN program. This program had several drawbacks:

(1) The transfer of data had to be done on a line that ran directly between the PDP and the IBM computers at a 300-baud rate. The 300-baud transfer rate meant that approximately 30 bytes of information per second could be transferred over our communications line. Normally, we collected a great number of data in any single experiment. Occasionally, as much as 15 min was required to transfer the data for one subject from the PDP computer to the IBM.

(2) Error checking of data was not possible. The software used on the IBM was in a "capture only" mode. That is, the computer simply captured everything that came over the communications line and stored it. Any

\footnotetext{
The authors may be written in care of the Department of Psychology, Trinity University, San Antonio, TX 78284.
}

noise induced into the lines could not easily be identified and corrected.

(3) A handshaking problem existed between the two computers. When communicating over an asynchronous line, our IBM computer operated in a half-duplex mode; that is; data traveled in only one direction at a time (in a full-duplex mode, data can travel in two directions simultaneously). In the half-duplex mode, after a line of data is sent to the host (IBM) and a carriage return/linefeed is pressed, the terminal (PDP) must wait for a "turnaround" sequence. This is the handshaking that the IBM uses in this general mode of communications. The FORTRAN program had to transmit a line of data and then wait for a response, repeating this for each line in the file. This caused some programming problems on the PDP and slowed down the communications process. Obviously, a better solution to the file-transfer problem had to be found.

In mid-1983, a file-transfer system was being distributed through the Digital Equipment Corporation Users Society (DECUS). This system (Kermit) permitted high-speed, error-free file transfer between various makes of computer equipment. Cruz and Catchings (1984a, 1984b) described their Kermit File Transfer Protocol in BYTE magazine. Although the article is somewhat technical, it is worthwhile for persons interested in computer networking. The main purpose of the article is to inform those who wish to develop and implement their own file-transfer system (FTS).

The Kermit system uses asynchronous communication lines to connect the computers in a network. These are serial lines typically used to connect peripheral devices and terminals to the host computer. At most institutions, an installation with a mainframe or minicomputer probably already has the physical requirements for using Kermit.

In order for Kermit to function, the program must be running on each computer involved in the transfer of data. In transferring a file from the PDP to the IBM, we run the Kermit program on the PDP, connect to the IBM system, $\log$ on, and run the program on the IBM. In this way, data files can be transferred in either direction intact and error free. 
The method used by Kermit to communicate between computers is one in which the original data set is broken into "packets," which have "checksums" associated with them. These checksums are used by Kermit on the receiving end to verify the correct transmission of the data. A reply packet is sent back to the transmitting system. If there is an error, Kermit automatically attempts to resend the information in which there was an error. Otherwise, Kermit transmits the next packet of data. This continues until the file(s) are correctly transferred or the transmission is aborted.

This FTS corrects most of the file-transfer problems that we have encountered in recent years. The use of Kermit speeds up the transfer rate and assures full error checking and correction. For computers that communicate with an IBM 370, a special "IBM Mode" is available on some versions of Kermit. This mode makes special allowance for the handshaking methods that are unique to the IBM protocol.

In our laboratory, fast and accurate file transfer is an important function. The PDP is used for data collection and $\mathrm{A} / \mathrm{D}$ conversion. The large IBM $370-3031$ is designed to perform large numbers of mathematical calculations and is, therefore, the ideal computer on which to perform the FFT. If we could not communicate (transfer data files) between the PDP and IBM computers, three alternatives would be available. First, the data could be reentered by hand into the IBM. This process would be very time consuming, and there would be the possibility of making dataentry errors. Second, we could develop a method of transferring data between the computers. This is not a viable alternative, since we have neither the time nor the staff to develop such a program. Third, we could return to the method used many years ago. For obvious reasons, this is not a reasonable alternative.
On the Trinity University campus, we are at present using or are in the process of installing Kermit on the following computers:

(1) IBM Mainframe 370-under CMS;

(2) DEC PDP-11/24 running RSX $11 \mathrm{M}$ version 4.0;

(3) IBM PC-DOS 2.0 or higher;

(4) Apple II+, Apple IIe, Apple IIc;

(5) DEC Pro 350 running single-user RSX; and

(6) Atari 800

Theoretically, once Kermit has been made operational on a computer, it can communicate with any other computer using Kermit. This permits the transfer of data files and source code (programs) from any computer to any other computer. Kermit works on asynchronous lines wired directly between computers, or over phone lines (which are serial asynchronous lines). Also, Kermit is user supported and is free for all computers (some installations that distribute kits may charge for documentation, labor, media, etc.). Users of the Kermit system are encouraged to send in new versions and modifications of the system to Columbia University, which is where the Kermit system originated and is the main distribution point for the system. The Kermit File Transfer Protocol is a very economical and effective tool for use in an environment that requires file transfer among computer systems.

\section{REFERENCES}

Cruz, F., \& Catchings, B. (1984a, June). Kermit: A file transfer protocol for universities, Part 1: Design considerations and specifications. BYTE, pp. 255-278.

Cruz, F., \& Catchings, B. (1984b, July). Kermit: A file transfer protocol for universities, Part 2: States and transitions, hueristic nules, and examples. BYTE, pp. 143-145, 400-403. 